\title{
Synthesis and characterization of novel
}

\section{poly(ethylene furanoate-co-ethylene vanillate) copolymers}

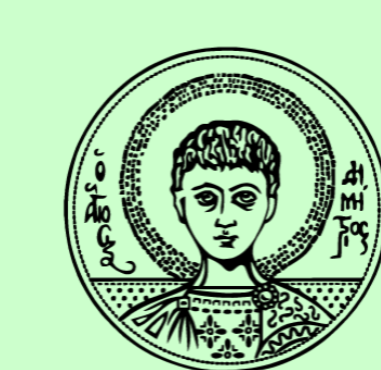

ARISTOTLE UNIVERSITY OF THESSALONIKI

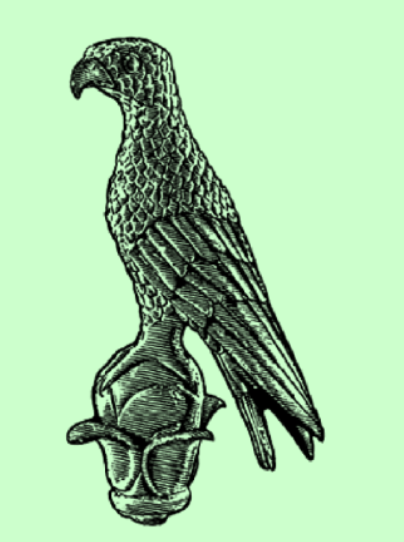

UNIVERSITY OF IOANNINA
${ }^{1}$ Evangelia Mpalla, ${ }^{1}$ Alexandra Zamboulis, ${ }^{1}$ Lazaros Papadopoulos, ${ }^{2}$ Eleftheria Xanthopoulou

${ }^{1}$ Zoi Terzopoulou, ${ }^{1}$ Dimitrios N. Bikiaris, ${ }^{2}$ George Z. Papageorgiou

[1] Department of Chemistry, Aristotle University of Thessaloniki, GR-541 24, Thessaloniki, Greece

[2] Chemistry Department, University of Ioannina, P.O. Box 1186, GR-45110, Ioannina, Greece

\section{Introduction}

Sustainable polymers \& Lignin

- Monomers based on renewable sources afford green polymers

- Lignin is the second most abundant natural polymer

- Source of aromatic monomers

Vanillic acid

- Produced by vanillin oxidation

- Biobased aromatic building block

- Poly(ethylene vanillate) has comparable thermal properties to PET
Poly(ethylene furanoate) (PEF)

- One of the most important biobased polymers

- Exceptional barrier properties $\left(\mathrm{O}_{2}\right.$ and $\left.\mathrm{CO}_{2}\right)$

- Superior thermal stability and lower melting temperature than PET

Our goals

- Prepare poly(ethylene furanoate)/poly(ethylene vanillate) copolymers (PEFV)

- Study the thermal behavior and stability

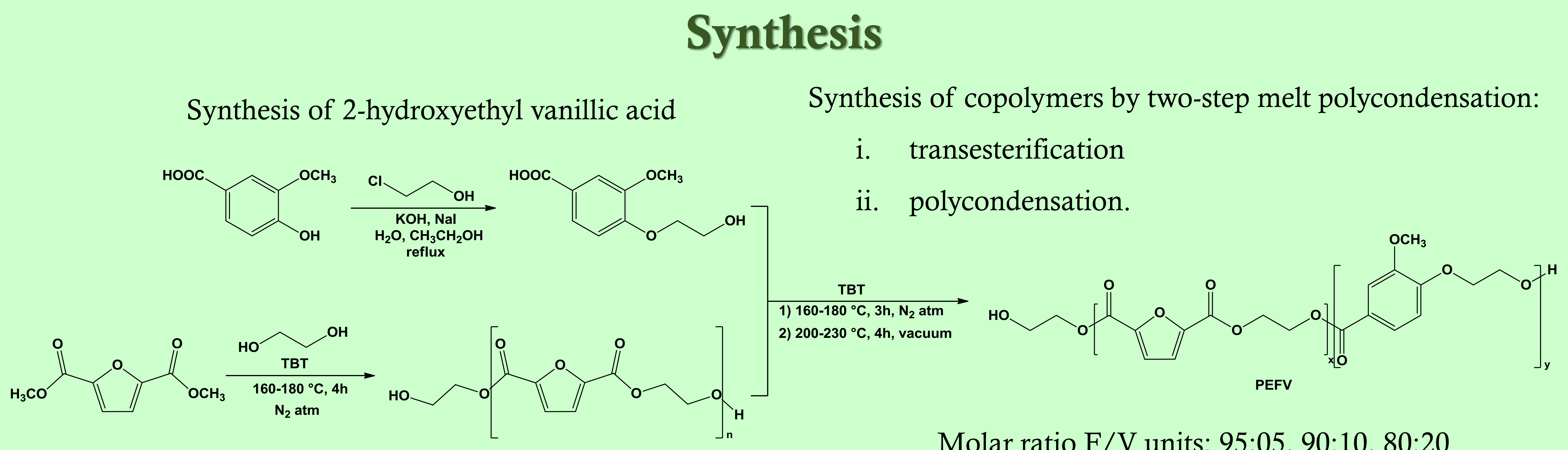

Synthesis of poly(ethylene 2,5-furandicarboxylate) oligomers

Molar ratio F/V units: 95:05, 90:10, 80:20

\section{Results}

NMR

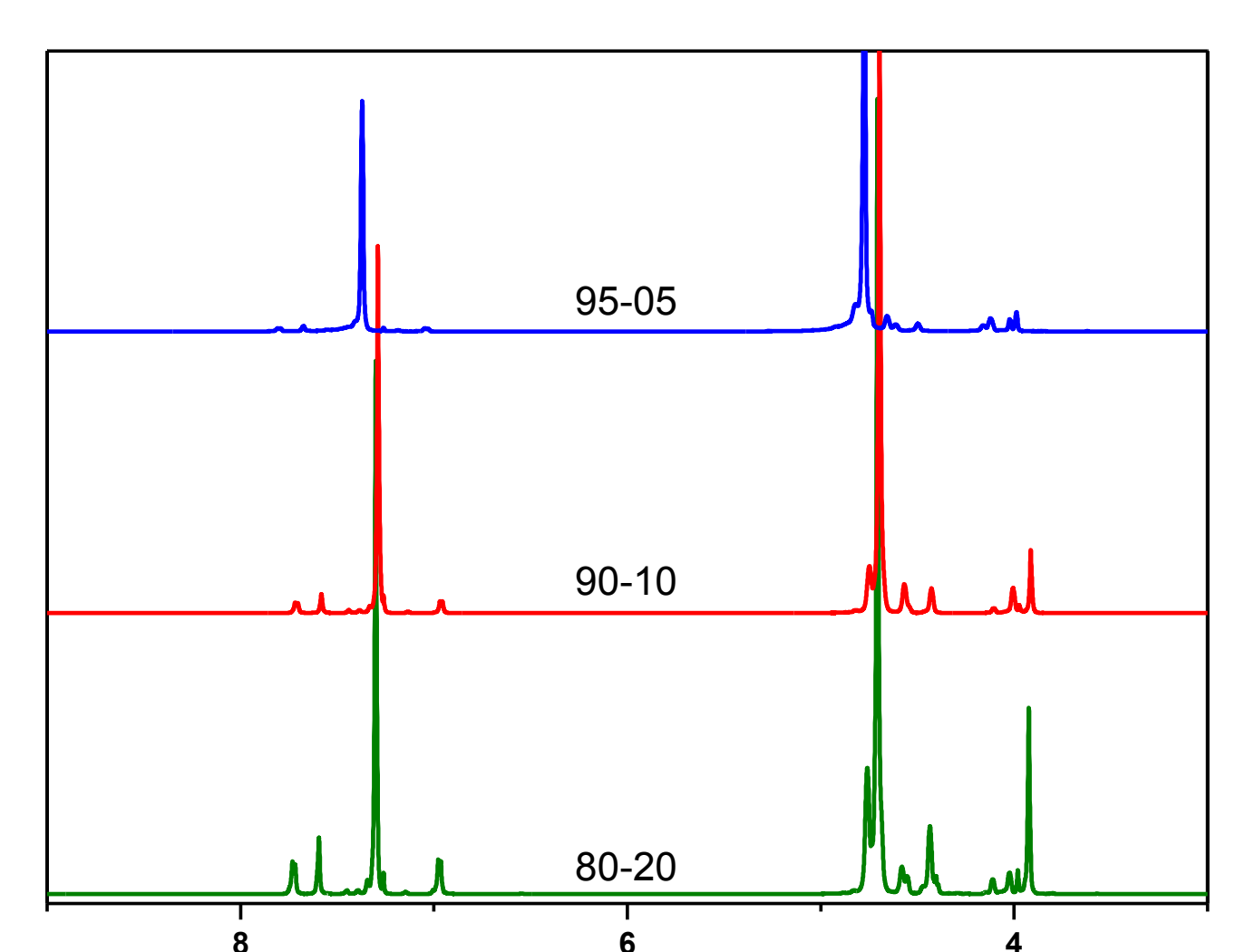

0
8
$8(\mathrm{ppm})$

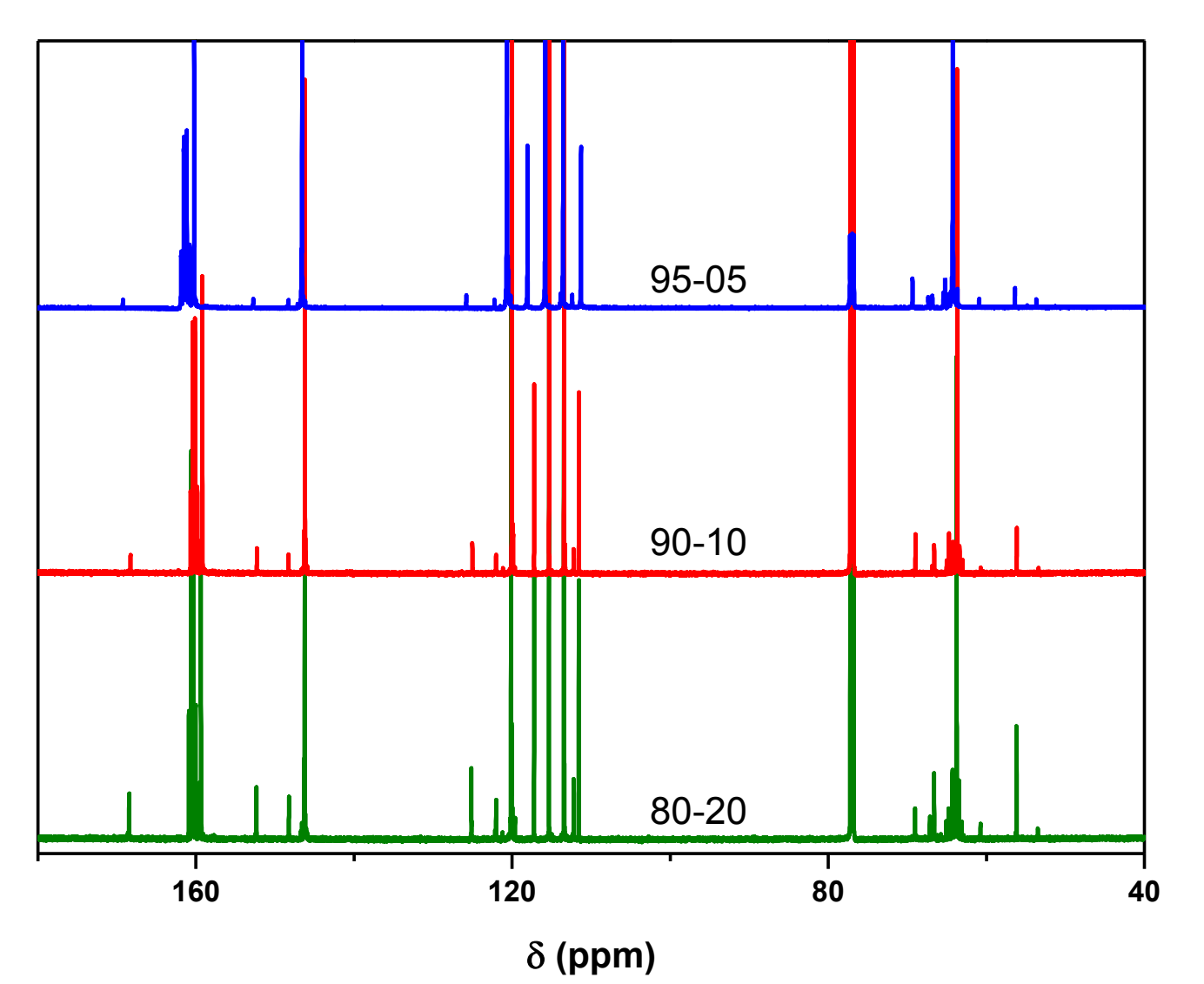

DSC

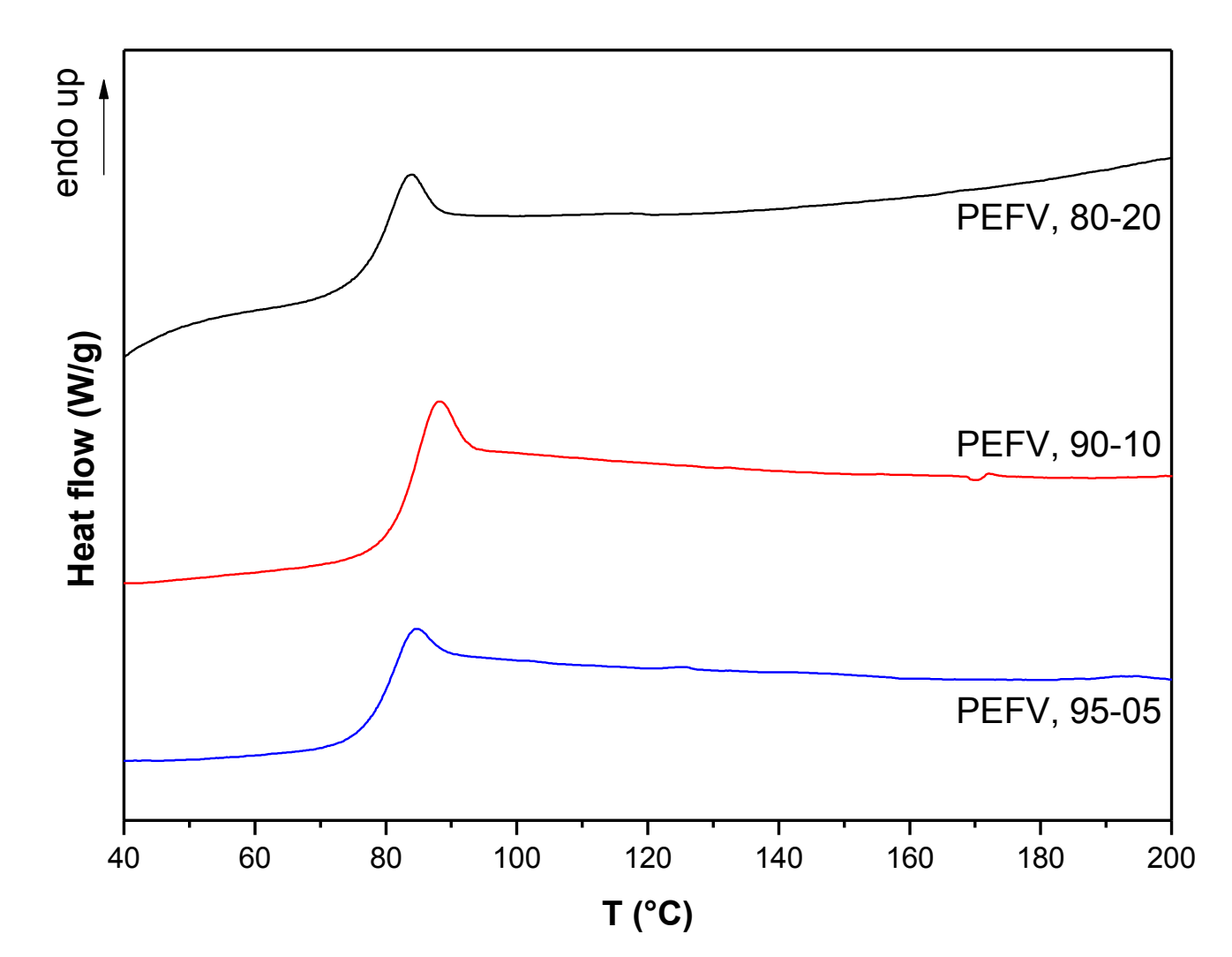

\begin{tabular}{|c|c|}
\multicolumn{2}{|c|}{$\mathrm{T}_{\mathrm{g}}\left({ }^{\circ} \mathrm{C}\right)$} \\
\hline PEFV 95-05 & 78 \\
PEFV 90-10 & 82 \\
PEFV 80-20 & 79 \\
\hline
\end{tabular}

$\mathrm{XRD}$
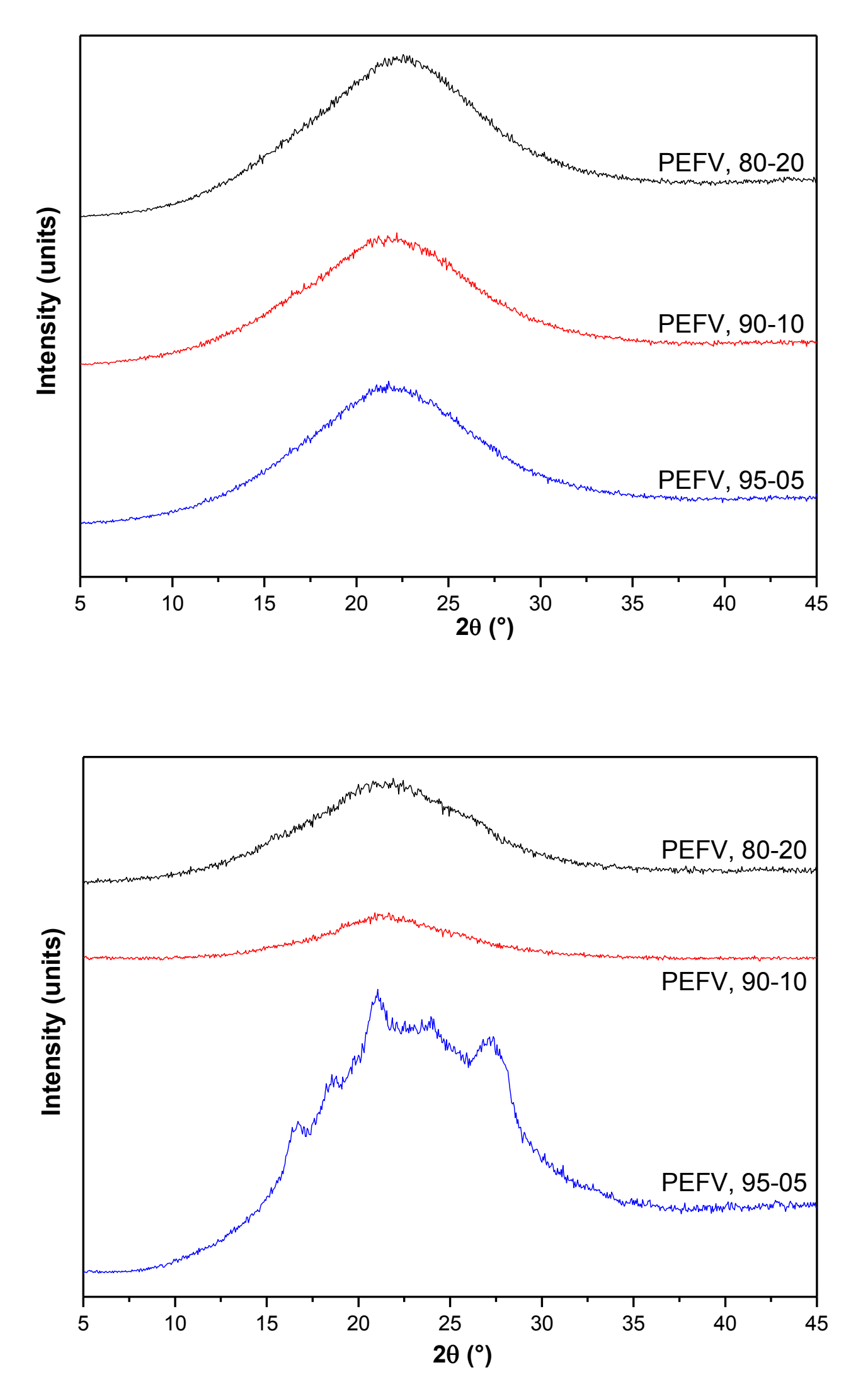

TGA
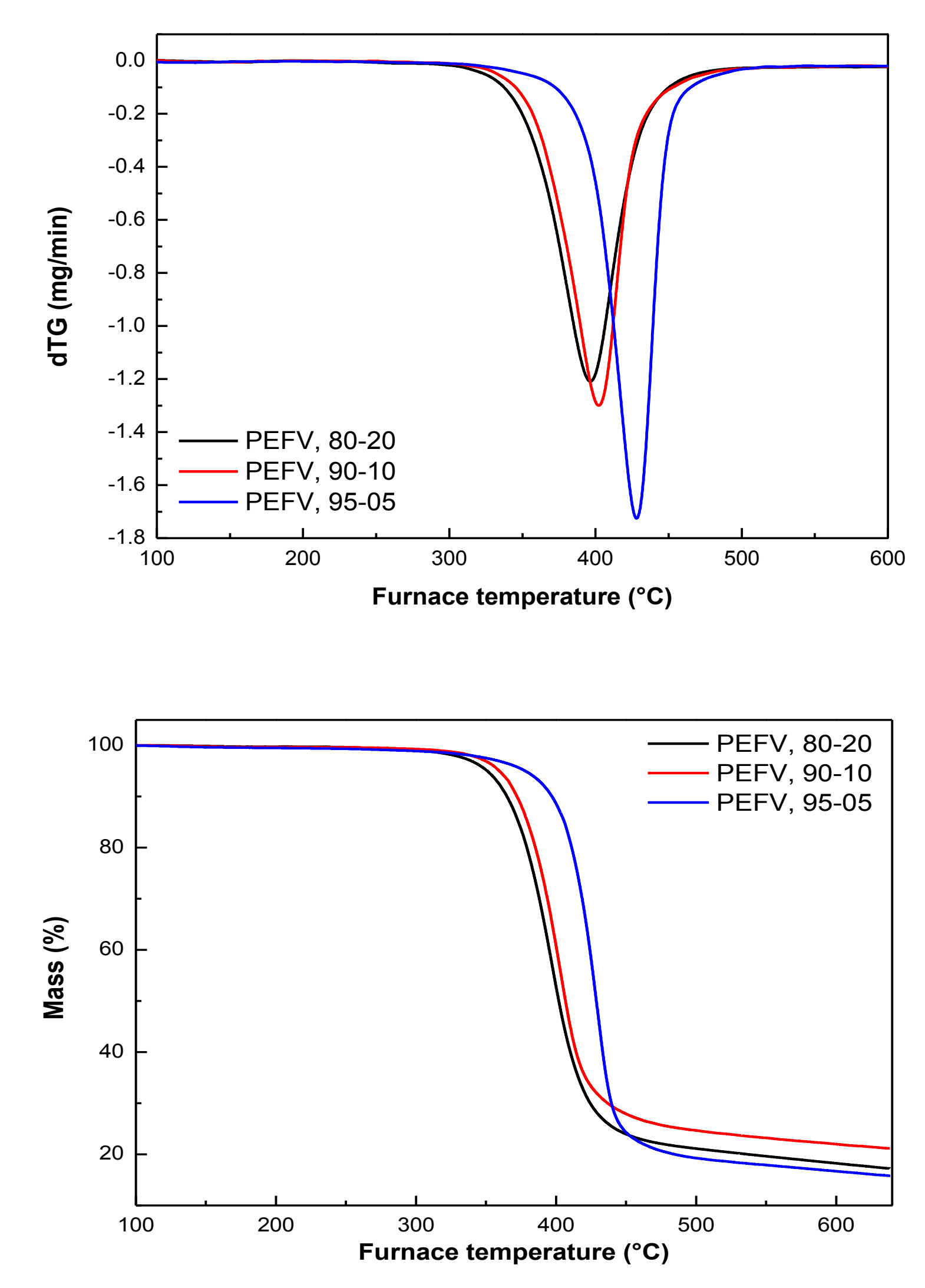

- NMR analysis confirms the successful synthesis and points to block copolymers.

- Copolymers were initially amorphous, but upon annealing $\left(1 \mathrm{~h}, \quad 100 \quad{ }^{\circ} \mathrm{C}\right)$ crystallinity increases.

- Crystallinity decreases with increasing vanillic content.

- Thermal stability decreases with increasing vanillic content.

\section{Conclusions}

$\checkmark$ PEFV block copolymers were successfully synthesized.

$\checkmark$ Composition is consistent with the feed ratio.

$\checkmark$ As received samples were completely amorphous.

$\checkmark \mathrm{T}_{\mathrm{g}}$ temperatures range around $80^{\circ} \mathrm{C}$.
The authors would like to acknowledge the Center of Interdisciplinary Research and Innovation of Aristotle University of Thessaloniki (CIRI-AUTH), Greece, for access to the Large Research Infrastructure and Instrumentation of the Nuclear Magnetic Resonance Laboratory at the Center for Research of the Structure of Matter in the Chemical Engineering Department. azamboulis@gmail.com dbic@chem.auth.gr 